\title{
Perovskite Oxide Nanocomposites for Thermoelectric Applications
}

\author{
S. Susithra ${ }^{1}$, Nithya Davis ${ }^{2}$, Anuradha M Ashok*² and V.K. Manoharan ${ }^{1}$ \\ \{ss.eee@psgtech.ac.in ${ }^{1}$, ama.fml@psgias.ac.in²,vkm.ece@psgtech.ac.in ${ }^{3}$ \} \\ Department of ECE, PSG College of Technology, Coimbatore ${ }^{1}$, Functional Materials Laboratory, \\ PSG Institute of Advanced Studies, Coimbatore ${ }^{2}$
}

\begin{abstract}
Oxides of transition elements with a perovskite structure is of significant interest due to their enhanced thermoelectric properties. Herein, we have synthesized $\mathrm{LaCoO} 3$ (LCO) and prepared composites with carbon nanofibers (CNF) in an effort to obtain increased power factor, thereby reducing lattice thermal conductivity $(\kappa L)$. $\mathrm{LaCoO} 3$ was prepared by Sol-Gel method using La and Co nitrates as precursors. The nanocomposites of LCO-CNF were prepared by traditional solid-state reaction. The phase purity of the samples was confirmed through XRD. The morphological characteristics of the pellets was studied through SEM analysis. Simultaneous electrical conductivity and seebeck measurements were performed in room temperature.
\end{abstract}

Keywords: Oxides, Perovskite, Thermoelectrics.

\section{Introduction}

One of the major problems that the world today is facing is that of energy crises. Only one-third of the energy from nuclear power plants, thermal power plant, automobiles and natural gas are consumed for various domestic and industrial activities. More than $60 \%$ of the energy from the above sources are wasted in the form of heat. Thus, there is a need for an alternative source of renewable and clean energy for the recovery of waste heat. Thermoelectrics enables the direct and reversible conversion of waste heat to useful electricity, thereby providing cleaner energy without any environmental impacts. Energy conversion through thermoelectrics depends on two major effects: Seebeck and Peltier effect.

In 1821, Seebeck observed that when two dissimilar materials are joined together and the junctions are held at different temperatures, a voltage difference developed that was proportional to the temperature difference (the Seebeck effect), as illustrated in Fig. 1.2. The ratio of the voltage developed in response to the temperature gradient is related to an intrinsic property of the materials called the Seebeck coefficient (S) or the thermopower. 


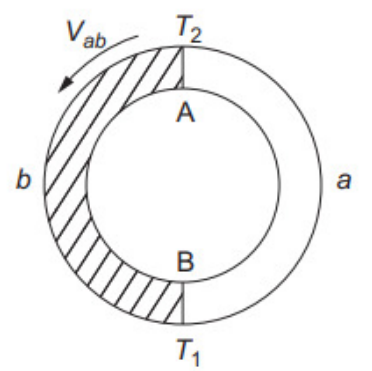

Fig 1.2: Seebeckeffect ${ }^{[1]}$

Later (in 1834), the Peltier effect was discovered, which states that when an electrical current is passed through a junction of two dissimilar materials, heat is either absorbed or given off at the junction, depending on the direction of the current, as shown in Fig. 1.3. This effect is due to the difference in Fermi energies of the two materials.

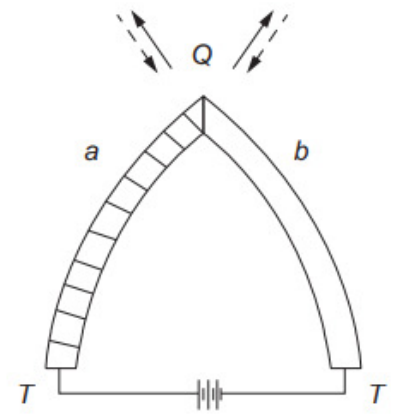

Fig 1.3: Peltier effect ${ }^{[1]}$

In the 1850s, William Thomson established the relationship between the Seebeck effect and the Peltier effect and predicted a third thermoelectric effect-the Thomson effect. The Seebeck effect provides the basic requirement for thermoelectric power devices and the Peltier effect provides the basis for many modern-day thermoelectric refrigeration devices.

A thermoelectric (TE) couple consists of a p-type and n-type semiconducting material connected through metallic pads. A thermoelectric module consists of array of these couples arranged electrically ion series and thermally in parallel. TE energy conversion uses the Peltier heat generated in the material to provide a temperature gradient with heat absorbed at the cold side, transferred through the TE material and rejected at the sink. This provides a refrigeration capability.

The TE performance of a material is given by the dimensionless thermoelectric figure of merit (ZT)

$Z T=\frac{S^{2} \sigma}{\kappa} T$

Where $\mathrm{S}$ is the Seebeckco-efficient in $\mathrm{V} / \mathrm{K}, \sigma$ is the electrical conductivity in $\mathrm{S} / \mathrm{m}, \kappa$ is the thermal conductivity in $\mathrm{W} / \mathrm{mK}$ and $\mathrm{T}$ is the absolute temperature in $\mathrm{K} . \mathrm{S}^{2} \sigma$ is the electrical power of the TE device which is termed as thermopower.To obtain a high figure of merit, it is necessary to have high power factor (higher $\sigma$ and relatively higher S) and lowered $\kappa$. 
The electrical conductivity is related to density of charge carriers (n) and mobility ( $\mu$ ) by,

$\sigma=n e \mu$.

Where $n$ is the density of charge carriers, $e$ is the electron charge and $\mu$ is the mobility of charge carriers.

The mobility is given by,

$\mu=\frac{e \tau}{m_{e}} \ldots \ldots \ldots$..... (1.3)

Where $\mu$ is the mobility of charge carriers, $m_{e}$ is the effective mass and $\tau$ is the mean scattering time between collision of charge carriers. The thermal conductivity is very large for metals. For semiconductors, the carriers must be excited thermally across the band gap for conduction to occur.

$\sigma \approx \sigma_{o} \exp \frac{E_{G}}{k_{B} T}$

The conductivity can occur due the contribution of electrons and holes given by,

$\sigma=n e \mu_{e}+p e \mu_{h}$

where $n$ and $p$ are electron and hole concentration respectively, and $\mu_{e}$ and $\mu_{h}$ are electron and hole concentration respectively.

Lowering the value of thermal conductivity can be achieved either by having a small gap to excite across $\left(\mathrm{E}_{\mathrm{G}}<\mathrm{k}_{\mathrm{B}} \mathrm{T}\right)$ or by having a very high carrier mobility.

The thermal conductivity is due to transfer of heat through the material by quantized vibration of lattice called phonons.

$\kappa=\kappa_{L}+\kappa_{E} \ldots \ldots \ldots . . .(1.6)$

where $\kappa_{\mathrm{L}}$ and $\kappa_{\mathrm{E}}$ are lattice and electronic contributions of thermal conductivity respectively.

The interdependency of thermal and electrical conductivity is established by the Widemann- Franz law and is given by

$\kappa_{E}=L_{o} \sigma T$

where $L_{o}$ is the Lorentz number. Increasing $\kappa$, results in an increased $\sigma$. But, for a higher ZT value, increasing $\sigma$ and decreasing $\kappa$ is necessary. This is one of the reason researchers are extensively working on materials and doping to obtain a higher value of ZT.

The conventional thermoelectric materials which include skutterides, tellurides and selenides are easily oxidized at high temperatures. They also have drawbacks such as: poor availability, high cost and toxicity. It is really challenging to obtain a thermoelectric material which is of low cost, stable at elevated temperature and non-toxic. Oxide based thermoelectric materials are of greater interest and being widely investigated by researchers. Among the oxide-based materials, $\mathrm{Na}_{\mathrm{x}} \mathrm{Co}_{2} \mathrm{O}_{4}, \mathrm{Ca}_{3} \mathrm{Co}_{4} \mathrm{O}_{9}$ and doped $\mathrm{ZnOare}$ most widely studied. Owing to their layered structure and multiple oxidation states, cobalt based oxides have relatively higher seebeck co-efficient. Another perovskite structured oxide, $\mathrm{LaCoO}_{3}$ (LCO) has been studied for TE applications ${ }^{[11]}$. $\mathrm{LaCoO}_{3}$ has a unique thermally induced spin transitions of Co. Also, it shows a behavioural change depending on temperature from n-type to p-type, thereby making it a widely studied thermoelectric material.

Our ultimate aim is to decrease $\kappa$ and increase power factor. Higher $\kappa$ value in TE materials is mainly due to higher lattice thermal conductivity $\left(\kappa_{\mathrm{L}}\right)$. Certain ways to decrease $\kappa$ without affecting $\sigma$ :

i. Nano-structuring, doping, creating oxygen vacancies, thereby decreasing $\kappa_{\mathrm{L}}$ through phonon scattering.

ii. Controlling the formation of double Schottky barrier and reducing the depletion region at the grain boundaries. This can be done by adding a conducting material to LCO.Carbon Nanofibers (CNF) has a unique $\sigma$, high specific surface area, 
great mechanical properties, zero bandgap, easy to disperse and can easily form composites with other oxides and semiconducting materials.

Henceforth, the present work aims at synthesizing LCO through sol-gel route using nitrates as precursors. The nanocomposite of LCO-CNF was prepared by traditional solid-state reaction. The pure LCO and the nanocomposite powders were pelletized using a hydraulic press to obtain circular pellets. The pellets were sintered at high temperature to obtain dense ceramics. X-Ray Diffractometry was carried out to check the phase purity of the pellets. The morphological study of the pellets was carried out using Scanning Electron Microscopy (SEM). The thermoelectric properties which includes the electrical conductivity andSeebeck co-efficient measurements were done.

\section{Experimental procedure}

\section{Synthesis of LCO:}

LCO was synthesized by sol-gel route using nitrates as precursors which is illustrated in fig 2.1. Stoichiometric ratio of Lanthanum nitrate and cobalt nitrate were dissolved in $100 \mathrm{~mL}$ water and kept for constant heat and stirring. Citric acid and PEG were added as chelating agents under constant stirring. The mixture was kept for stirring for 14 hours at $180^{\circ} \mathrm{C}$. The obtained gels were ground to fine powders and calcined at $900^{\circ} \mathrm{C}$ for 5 hours.

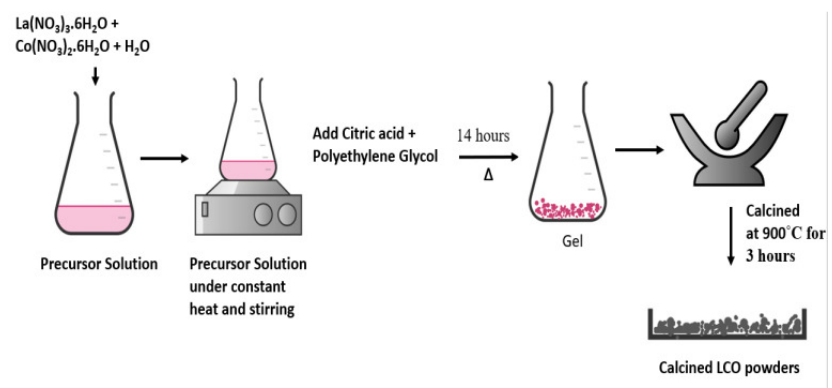

Fig 2.1: Synthesis of LCO through sol-gel route

The LCO-CNF nanocomposite was prepared by mechanical milling. An optimum amount of LCO and different weight percentage of CNF were weighed and a homogenous mixture of the same was achieved by the traditional solid-state reaction. The calcined pure LCO powders and the nanocomposites obtained by mechanical milling were pressed into pellets by using a hydraulic press at a pressure of $10 \mathrm{MPa}$. Circular pellets of pure LCO and LCO-CNF nanocomposite were obtained using hydraulic press which were further sintered at high temperature for 6 hours. The sintered pellets were subjected to XRD to check phase purity, SEM to study the morphological characteristics and simultaneous Seebeck co-efficient and electrical conductivity measurements to understand the thermoelectric properties. 


\section{Results And Discussions}

Combined Thermo-Gravimetry and Differential Scanning Calorimetry (TG-DSC) analysis:

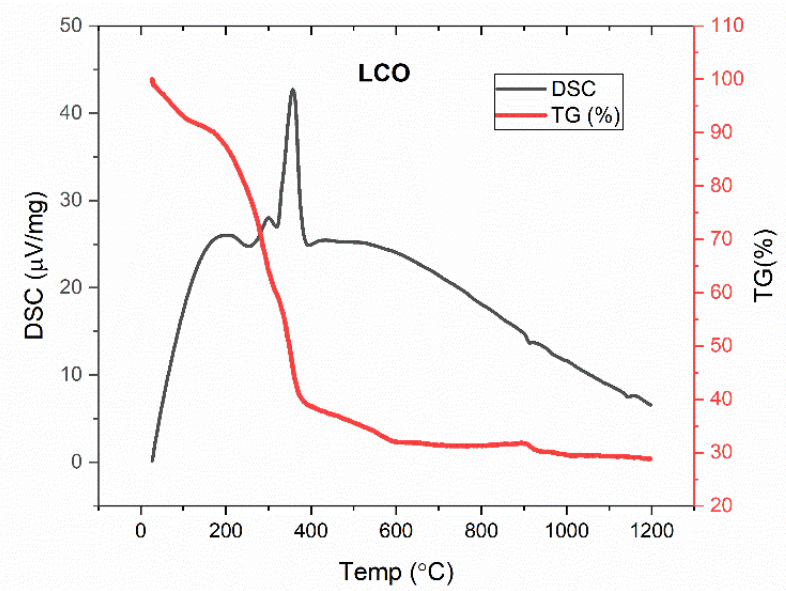

Fig 3.1: TG-DSC plot for uncalcined LCO powders

Fig 3.1 gives the TG-DSC plot for uncalcined LCO powders. The first drop in the TG plot at $350^{\circ} \mathrm{C}$ is due to the evolution of water from the sample. The second drop at $600^{\circ} \mathrm{C}$ is due to the evolution of nitrates from the sample (Since, we used nitrates as precursors). After the drop at $900^{\circ} \mathrm{C}$, the graph is linear without any loss. Therefore, the calcination temperature is determined to be $900^{\circ} \mathrm{C}$. From the DSC plot, it is evident that the reaction is exothermic.

\section{X-Ray Diffraction (XRD):}

Fig 3.2 shows the XRD plot for pure LCO. The peaks in the XRD plot are distinct and sharp indicating that the sample is highly crystalline. All the peaks were indexed by PCPDS card no. 48-0123 of $\mathrm{LaCoO}_{3}$. There are no secondary peaks in the XRD plot which indicated the pellets are phase pure. However, no peaks corresponding to carbon was observed in diffractograms taken from LCO-CNF nanocomposite. This is due to the very small concentration of CNF present in the nanocomposite. 


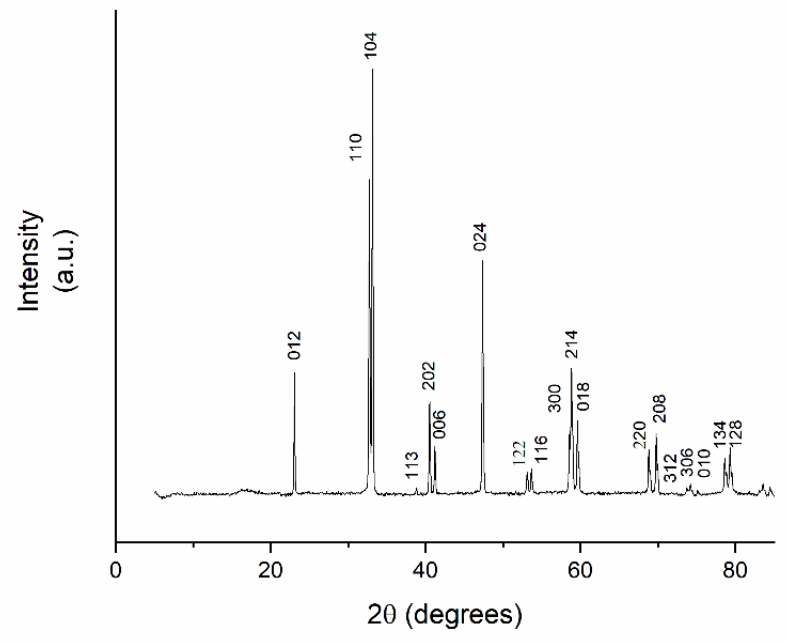

Fig 3.2: XRD plot of the sintered pellet

Scanning Electron Microscopy (SEM):

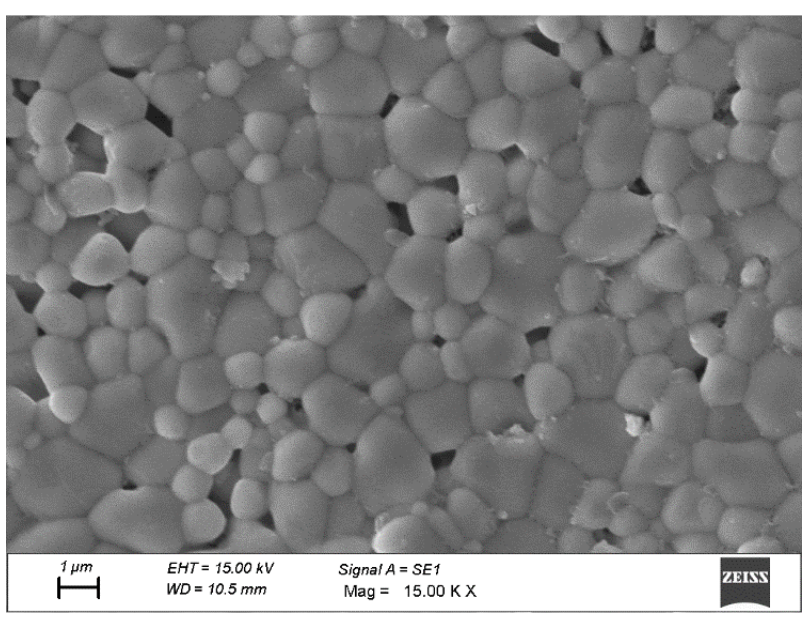

Fig 3.3: SEM image of pure LCO 


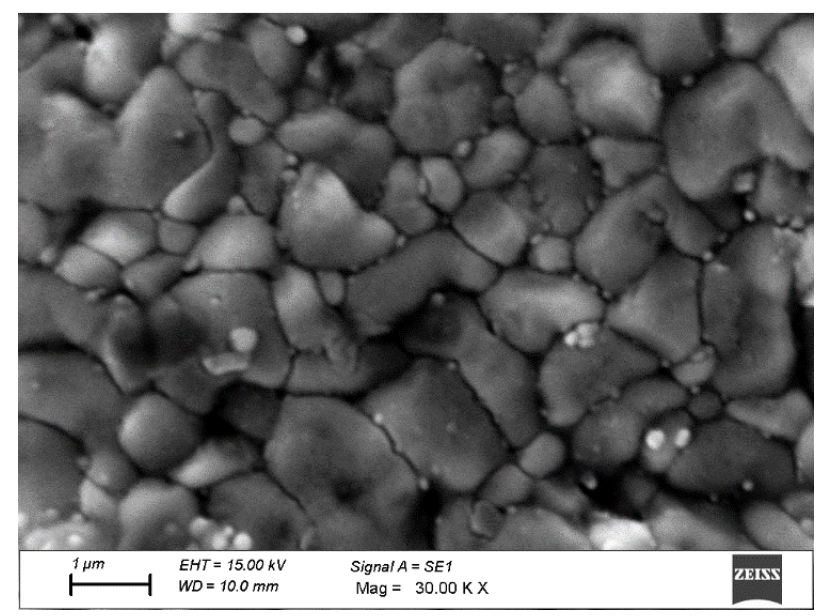

Fig 3.4: SEM image of LCO-CNF nanocomposite

Fig 3.3 and 3.4 gives theScanning Electron Micrographs of LCO and LCO-CNF nanocomposite respectively. From the figure, it can be seen that the pellets are dense sintered ceramics, indicating the effect of high temperature sintering. The pellets were also found to have distinct grains and grain boundaries. Also, the pellets do not have any agglomeration which indicates that CNF is completely dispersed and are well interacted with the LCO. The distinct grain and grain boundaries greatly minimize phonon scattering thereby effectively reducing thermal conductivity without significantly affecting electrical conductivity.

\section{Conclusion}

The $\mathrm{LaCoO}_{3}$ powders obtained from sol-gel route were found be highly crystalline and phase pure without any secondary phases after analysis using XRD. The $\mathrm{LaCoO}_{3}-\mathrm{CNF}$ nanocomposites were prepared by traditional solid-state method and pelletized. From the SEM images, it is evident that the pellets are dense sintered with distinct grain and grain boundaries that would reduce the thermal conductivity without affecting electrical conductivity. The presence of CNF was confirmed using HRTEM images. The LCO-CNF nanocomposite would lead to a better thermoelectric property.

\section{References}

[1] Ji, L., 2018. Metal oxide-based thermoelectric materials. In Metal Oxides in Energy Technologies (pp. 49-72). Elsevier.

[2] Nagira, T., Ito, M., Katsuyama, S., Majima, K. and Nagai, H., 2003. Thermoelectric properties of (Na1- yMy) xCo2O4 $(\mathrm{M}=\mathrm{K}, \mathrm{Sr}, \mathrm{Y}, \mathrm{Nd}, \mathrm{Sm}$ and $\mathrm{Yb} ; \mathrm{y}=0.01 \sim 0.35)$. Journal of alloys and compounds, 348(1-2), pp.263-269.

[3] Ito, M. and Furumoto, D., 2008. Microstructure and thermoelectric properties of NaxCo2O4/Ag composite synthesized by the polymerized complex method. Journal of alloys and compounds, 450(1-2), pp.517-520. 
[4] Wang, Y., Sui, Y., Wang, X., Su, W. and Liu, X., 2010. Enhanced high temperature thermoelectric characteristics of transition metals doped $\mathrm{Ca} 3 \mathrm{Co} 4 \mathrm{O} 9+\delta$ by cold high-pressure fabrication. Journal of Applied Physics, 107(3), p.033708.

[5] Madre, M.A., Rasekh, S., Torres, M.A., Diez, J.C. and Sotelo, A., 2018. Improving bulk Ca3Co4O9 thermoelectric materials through $\mathrm{Zr}$ doping. Advances in Applied Ceramics, 117(3), pp.142-146.

[6] He, T., Chen, J., Calvarese, T.G. and Subramanian, M.A., 2006. Thermoelectric properties of La1$\mathrm{xAxCoO} 3(\mathrm{~A}=\mathrm{Pb}, \mathrm{Na})$. Solid state sciences, 8(5), pp.467-469.

[7] Feng, X., Fan, Y., Nomura, N., Kikuchi, K., Wang, L., Jiang, W. and Kawasaki, A., 2017. Graphene promoted oxygen vacancies in perovskite for enhanced thermoelectric properties. Carbon, 112, pp.169-176.

[8] Salah, N., Baghdadi, N., Alshahrie, A., Saeed, A., Ansari, A.R., Memic, A. and Koumoto, K., 2019. Nanocomposites of $\mathrm{CuO} / \mathrm{SWCNT}$ : promising thermoelectric materials for mid-temperature thermoelectric generators. Journal of the European Ceramic Society, 39(11), pp.3307-3314.

[9] Rahman, J.U., Van Du, N., Nam, W.H., Shin, W.H., Lee, K.H., Seo, W.S., Kim, M.H. and Lee, S., 2019. Grain Boundary Interfaces Controlled by Reduced Graphene Oxide in Nonstoichiometric SrTiO 3- $\delta$ Thermoelectrics. Scientific reports, 9(1), pp.1-12.

[10] Wu, C., Li, J., Fan, Y., Xing, J., Gu, H., Zhou, Z., Lu, X., Zhang, Q., Wang, L. and Jiang, W., 2019. The effect of reduced graphene oxide on microstructure and thermoelectric properties of Nb-doped A-site-deficient SrTiO3 ceramics. Journal of Alloys and Compounds, 786, pp.884-893.

[11] Muraleedharan, S., Davis, N., Althaf, R., Singh, A. and Ashok, A.M., 2020. Exploring the thermoelectric behavior of intrinsic and defect induced $\mathrm{LaCoO} 3$ with selected alkaline earth metals. Journal of Alloys and Compounds, p.157507.

[12] Zhang, B., Yu, C. and Li, Z., 2020. Enhancing the Electrochemical Properties of LaCoO 3 by SrDoping, rGO-Compounding with Rational Design for Energy Storage Device. Nanoscale Research Letters, 15(1), pp.1-13.

[13] Yan, Z., Sun, H., Chen, X., Fu, X., Chen, C., Cheng, F. and Chen, J., 2018. Rapid low-temperature synthesis of perovskite/carbon nanocomposites as superior electrocatalysts for oxygen reduction in Zn-air batteries. Nano Research, 11(6), pp.3282-3293.

[14] Aamir, M., Bibi, I., Ata, S., Majid, F., Kamal, S., Alwadai, N., Sultan, M., Iqbal, S., Aadil, M. and Iqbal, M., 2020. Graphene oxide nanocomposite with $\mathrm{Co}$ and $\mathrm{Fe}$ doped $\mathrm{LaCrO} 3$ perovskite active under solar light irradiation for the enhanced degradation of crystal violet dye. Journal of Molecular Liquids, p.114895.

[15] Ovik, R., Long, B.D., Barma, M.C., Riaz, M., Sabri, M.F.M., Said, S.M. and Saidur, R., 2016. A review on nanostructures of high-temperature thermoelectric materials for waste heat recovery. Renewable and sustainable energy reviews, 64, pp.635-659.

[16] Lv, H.Y., Liu, H.J., Shi, J., Tang, X.F. and Uher, C., 2013. Optimized thermoelectric performance of Bi 2 Te 3 nanowires. Journal of Materials Chemistry A, 1(23), pp.6831-6838.

[17] Heremans, J.P., Jovovic, V., Toberer, E.S., Saramat, A., Kurosaki, K., Charoenphakdee, A., Yamanaka, S. and Snyder,

G.J., 2008. Enhancement of thermoelectric efficiency in PbTe by distortion of the electronic density of states. Science, 321(5888), pp.554-557.

[18] Yu, B., Zebarjadi, M., Wang, H., Lukas, K., Wang, H., Wang, D., Opeil, C., Dresselhaus, M., Chen, G. and Ren, Z., 2012. Enhancement of thermoelectric properties by modulation-doping in silicon germanium alloy nanocomposites. Nano letters, 12(4), pp.2077-2082.

[19] Cho, J.Y., Siyar, M., Jin, W.C., Hwang, E., Bae, S.H., Hong, S.H., Kim, M. and Park, C., 2019. Electrical Transport and Thermoelectric Properties of SnSe-SnTe Solid Solution. Materials, 12(23), p.3854.

[20]Zhu Z, Zhang Y, Song H, Li X-J (2018) Enhancement of thermoelectric performance of Cu1.98Se by $\mathrm{Pb}$ doping. Appl Phys A Mater Sci Process. 
[21]Zhu, H., Mao, J., Li, Y., Sun, J., Wang, Y., Zhu, Q., Li, G., Song, Q., Zhou, J., Fu, Y. and He, R., 2019. Discovery of TaFeSb-based half-Heuslers with high thermoelectric performance. Nature communications, 10(1), pp.1-8. 